\title{
THE 2-CHARACTER TABLE DOES NOT DETERMINE A GROUP
}

\author{
KENNETH W. JOHNSON AND SURINDER K. SEHGAL
}

(Communicated by Ronald M. Solomon)

\begin{abstract}
Frobenius had defined the group determinant of a group $G$ which is a polynomial in $n=|G|$ variables. Formanek and Sibley have shown that the group determinant determines the group. Hoehnke and Johnson show that the 3-characters (a part of the group determinant) determine the group. In this paper it is shown that the 2-characters do not determine the group. If we start with a group $G$ of a certain type then a group $H$ with the same 2-character table must form a Brauer pair with $G$. A complete description of such an $H$ is available in Comm. Algebra 9 (1981), 627-640.
\end{abstract}

\section{INTRODUCTION}

The group determinant was first introduced in 1896 by Frobenius [3]. It was the problem of how this determinant factorizes which led him to define characters for an arbitrary finite group $G$. It is a natural question to consider whether $G$ is determined by its group determinant, but it appears that this question was not raised until 1986 (Johnson [7]). In fact the group determinant contains sufficient information to determine a group. This was shown in 1990 by Formanek and Sibley [4], and recently an elementary proof has appeared by Mansfield [9]. In [3] there were also introduced functions $\chi^{(k)}: G^{k} \rightarrow \mathbb{C}$ which correspond to a character $\chi$ of $G, k=1,2, \ldots$. These were named $k$-characters in [8]. It follows from [3] that if $\left\{\chi_{i}\right\}, 1 \leq i \leq m$, is the set of distinct irreducible characters of $G$ then $\left\{\chi_{i}^{(k)}, 1 \leq k \leq \operatorname{deg}\left(\chi_{i}\right), 1 \leq i \leq m\right\}$ determines the group determinant of $G$, and hence $G$.

Recently it has been announced by Hoehnke and Johnson [5] that the 3character of the regular representation, or equivalently the knowledge of the $1-, 2-$, and 3-characters corresponding to all the irreducible characters of $G$, is sufficient to determine $G$. If $\chi$ is a character of $G$, the 1-, 2-, and 3-characters

Received by the editors May 14, 1991.

1991 Mathematics Subject Classification. Primary 20C15; Secondary 20B20, $20 \mathrm{~B} 10$.

Key words and phrases. Group determinant, character table, Brauer pairs.

The first author was partially supported by the International Mathematical Research Institute at The Ohio State University. 
corresponding to $\chi$ are defined as follows:

$$
\begin{aligned}
& \chi^{(1)}(g)=\chi(g), \quad g \in G, \\
& \chi^{(2)}(g, h)=\chi(g) \chi(h)-\chi(g h), \quad g, h \in G, \\
& \chi^{(3)}(g, h, k)=\chi(g) \chi(h) \chi(k)-\chi(g) \chi(h k)-\chi(h) \chi(g k) \\
& \quad-\chi(k) \chi(g h)+\chi(g h k)+\chi(g k h), \quad g, h, k \in G .
\end{aligned}
$$

Thus an answer is provided to the question of Brauer in [1] as to which information in addition to the (ordinary) character table of a group is sufficient to determine a group.

There remains the question of whether a group can be determined by its 2characters. In [8] a 2-character table of a finite group $G$ is defined. If the set of irreducible characters of $G$ is $\left\{\chi_{1}, \ldots, \chi_{m}\right\}$ then two types of "degenerate" 2-characters are defined:

$$
\begin{aligned}
& \text { (i) } \chi_{i} \circ \chi_{j}(g, h)=\chi_{i}(g) \chi_{j}(h)+\chi_{i}(h) \chi_{j}(g), \quad 1 \leq i<j \leq m, \\
& \text { (ii) } \chi_{i}^{(2,+)}(g, h)=\chi_{i}(g) \chi_{i}(h)+\chi_{i}(g h), \quad 1 \leq i \leq m .
\end{aligned}
$$

The 2-character table of $G$ then consists of the $\chi_{i}^{(2)}$, where $\operatorname{deg}\left(\chi_{i}\right) \geq 2$, and the degenerate 2-characters described in (1.2). It is shown in [8] that orthogonality relations hold among these 2-characters.

A consequence of Theorem 2.1 of this work is that there exist pairs of nonisomorphic groups with the same 2-character table, an explicit example of such a pair being two groups of order $624 \cdot 625$. It follows that the 2-characters are not sufficient to determine a group.

We remark that $G$ and $H$ have the same 2-character tables if and only if there exists a map $\psi: G \rightarrow H$ and a correspondence $\chi_{i} \leftrightarrow \mu_{i}$ between the irreducible characters of $G$ and $H$ such that for each generalized 2-character $\nu$ of $G$ (see above)

$$
\nu(g, h)=\tau(\psi(g), \psi(h)),
$$

where $\tau$ is the 2-character of $H$ which corresponds to $\nu$ under the correspondence induced by $\chi_{i} \leftrightarrow \mu_{i}$.

Throughout the paper it is assumed that all characters are complex characters.

\section{Doubly TRANSITIVE SOlVABLE FrobeniUs GROUPS}

In [2] it is shown that if $G$ is a doubly transitive solvable permutation group and if the group $H$ has the same ordinary character table as $G$ then $H$ must also be a doubly transitive solvable permutation group, and $\{G, H\}$ is a Brauer pair. Moreover, the classification of Huppert [6] may be used to show that, apart from exceptional cases in which the character table of $G$ determines $G$ uniquely, $G$ and $H$ must be subgroups of $F S\left(p^{n}\right)$, the group of semilinear maps of the finite field $F=G F\left(p^{n}\right)$. We prove the following theorem, which depends on results in [2].

Theorem 2.1. Let $G$ be a doubly transitive solvable Frobenius group. Then the following are equivalent:

(a) The groups $G$ and $H$ form a Brauer pair.

(b) The groups $G$ and $H$ have the same 2-character tables. 
Proof. We first prove that $(\mathrm{a}) \Rightarrow(\mathrm{b})$. Suppose that $G$ is a doubly transitive Frobenius group and that there is a group $H$ not isomorphic to $G$ such that $\{G, H\}$ is a Brauer pair. In [2] it is shown that $G$ and $H$ must be subgroups of $F S\left(p^{n}\right)$ of the following form. Let $\sigma$ denote the Frobenius automorphism $\left(\begin{array}{c}x \\ x^{p}\end{array}\right)$ of $F, \bar{\omega}$ denote the map $\left(\begin{array}{c}x \\ x \omega\end{array}\right)$ of $F$ (for any $w \in F$ ), and $n^{*}$ denote the map $\left(\begin{array}{c}x \\ x+n\end{array}\right)$ (for any $n \in F$ ). From now on let us pick $\omega$ to be a generator of the multiplicative group of $F$. Let $N=\left\langle n^{*} ; n \in F\right\rangle$ be the subgroup of $F S\left(p^{n}\right)$ isomorphic to the additive group of $F$. Then

$$
\begin{array}{ll}
G \simeq G_{0} \ltimes N, & \text { where } G_{0}=\left\langle\bar{\omega}^{k}, \sigma^{v} \bar{\omega}^{i}\right\rangle, \\
H \simeq H_{0} \ltimes N, & \text { where } H_{0}=\left\langle\bar{\omega}^{k}, \sigma^{v} \bar{\omega}^{j}\right\rangle
\end{array}
$$

with $k /\left(p^{n}-1\right), v k=n,(k, i)=(k, j)=1$, and such that $p^{v}$ has order $k$ modulo $k\left(p^{v}-1\right)$.

Lemma 2.2. Suppose $(k, i)=1$ and $(k, j)=1$. Then a prime $q$ cin be chosen such that $q \nmid p^{n}-1$ and $j \equiv i q$ modulo $k$.

Proof. Let

$$
x \equiv j i^{-1} \bmod k,
$$

where $i^{-1}$ is the inverse of $i$ in $\mathbb{Z}_{k}$. Then $x=a+k t, a=j \cdot i^{-1}$. Thus any element of the arithmetic progression $\{a+k t ; t=1,2, \ldots\}$ is a solution of (2.1) and by Dirichlet's theorem a prime solution $q$ may be chosen such that $q \nmid p^{n}-1$. Then $j \equiv i q \bmod k$.

Lemma 2.3. Define the map $\theta: G_{0} \rightarrow H_{0}$ by

$$
\theta\left(\bar{\omega}^{k}\right)=\bar{\omega}^{k q}, \quad \theta\left(\sigma^{v} \bar{\omega}^{i}\right)=\sigma^{v} \bar{\omega}^{i q},
$$

where $q$ is a prime satisfying the conditions of Lemma 2.2. Then $\theta$ extends to an isomorphism from $G_{0}$ to $H_{0}$, such that $\theta\left(\sigma^{v t} \bar{\omega}^{r}\right)=\sigma^{v t} \bar{\omega}^{r q}$ whenever $\sigma^{v t} \bar{\omega}^{r}$ lies in $G_{0}$.

Proof. It is clear that $\theta\left(\bar{\omega}^{k}\right)$ lies in $H_{0}$. Now

$$
\theta\left(\sigma^{v} \bar{\omega}^{i}\right)=\sigma^{v} \bar{\omega}^{i q}=\sigma^{v} \bar{\omega}^{j+k \lambda}, \quad \lambda \in \mathbb{Z},
$$

since $q \equiv j \bmod k$ and thus

$$
\theta\left(\sigma^{v} \bar{\omega}^{i}\right)=\left(\sigma^{v} \bar{\omega}^{j}\right)\left(\bar{\omega}^{k \lambda}\right) \text { lies in } H_{0} .
$$

The elements of $G_{0}$ may be described uniquely as those of the form

$$
\left(\sigma^{v} \omega^{i}\right)^{t} \bar{\omega}^{k \lambda}, \quad 0 \leq t \leq \frac{n}{v}, 1 \leq \lambda \leq \frac{p^{n}-1}{k} .
$$

For suppose $\left(\sigma^{v} \bar{\omega}^{i}\right)^{t} \bar{\omega}^{k \lambda}=\left(\sigma^{v} \omega^{i}\right)^{t^{\prime}} \bar{\omega}^{k \lambda^{\prime}}$ with $0 \leq t^{\prime} \leq t \leq n / v$ and $1 \leq \lambda, \lambda^{\prime} \leq\left(p^{n}-1\right) / k$. It follows that $\left(\sigma^{v} \bar{\omega}^{i}\right)^{t-t^{\prime}}=\bar{\omega}^{k\left(\lambda^{\prime}-\lambda\right)}$, i.e.,

$$
\sigma^{v\left(t-t^{\prime}\right)} \bar{\omega}^{s}=\bar{\omega}^{k\left(\lambda^{\prime}-\lambda\right)} \text { for some } s
$$

(using Lemma 1.2(vi) in [2]). Therefore $t-t^{\prime}=0$, and hence $\lambda-\lambda^{\prime}=0$. Thus the elements in (2.2) are all distinct, and by counting must form all the elements of $G_{0}$. 
Now define

$$
\begin{aligned}
\theta\left(\left(\sigma^{v} \bar{\omega}^{i}\right)^{t} \bar{\omega}^{k \lambda}\right) & =\left(\theta\left(\sigma^{v} \bar{\omega}^{i}\right)\right)^{t}\left(\theta\left(\bar{\omega}^{k}\right)\right)^{\lambda}=\left(\sigma^{v} \bar{\omega}^{i q}\right)^{t} \bar{\omega}^{k q \lambda} \\
& =\sigma^{v t} \bar{\omega}^{i q\left(\left(p^{v t}-1\right) /(p-1)\right)+k q \lambda}
\end{aligned}
$$

again using Lemma 1.2(vi) in [2]. Note that since

$$
\left(\sigma^{v} \bar{\omega}^{i}\right)^{t} \bar{\omega}^{k \lambda}=\sigma^{v t} \bar{\omega}^{i\left(\left(p^{v t}-1\right) /(p-1)\right)+k \lambda}
$$

it follows that $\theta\left(\sigma^{v t} \bar{\omega}^{r}\right)=\sigma^{v t} \bar{\omega}^{r q}$ whenever $\sigma^{v t} \bar{\omega}^{r}$ lies in $G_{0}$.

Therefore for elements $\sigma^{v t} \bar{\omega}^{r}$ and $\sigma^{v t^{\prime}} \bar{\omega}^{r^{\prime}}$ of $G_{0}$ we obtain

$$
\begin{aligned}
\theta\left[\left(\sigma^{v t} \bar{\omega}^{r}\right)\left(\sigma^{v t^{\prime}} \bar{\omega}^{r^{\prime}}\right)\right] & =\theta\left[\sigma^{v\left(t+t^{\prime}\right)} \bar{\omega}^{r p^{v t^{\prime}}+r^{\prime}}\right] \quad(\text { using Lemma 1.2(v) in [2]) } \\
& =\sigma^{v\left(t+t^{\prime}\right)} \bar{\omega}^{q\left(r p^{v t^{\prime}}+r^{\prime}\right)}=\sigma^{v t} \bar{\omega}^{q r} \sigma^{v t^{\prime}} \bar{\omega}^{q r^{\prime}} \\
& =\theta\left(\sigma^{v t} \bar{\omega}^{r}\right) \theta\left(\sigma^{v t^{\prime}} \bar{\omega}^{r^{\prime}}\right)
\end{aligned}
$$

Hence $\theta$ is a homomorphism of $G_{0}$ into $H_{0}$.

Suppose $\theta\left(\sigma^{v t} \bar{\omega}^{r}\right)=e$. Then $\sigma^{v t} \bar{\omega}^{r q}=e$, and thus $\sigma^{v t}=e$ and $\bar{\omega}^{r q}=e$ since $\langle\sigma\rangle \cap\langle\bar{\omega}\rangle=\{e\}$. Hence $\operatorname{ker} \theta=\{e\}$ and Lemma 2.3 is proved.

Now define the map $\psi: G \rightarrow H$ by

$$
\psi\left(g_{0} n^{*}\right)=\theta\left(g_{0}\right)\left(n^{q}\right)^{*}, \quad n^{*} \in N, g_{0} \in G_{0} .
$$

We will show that $\psi$ induces an identification of the 2-character tables of $G$ and $H$.

In [2] the character table of $G$ is determined (and is the same as that of $H$ ). It consists of characters $\chi_{1}, \ldots, \chi_{l}$ which are obtained from the irreducible characters $\bar{\chi}_{1}, \ldots, \bar{\chi}_{l}$ of $G$ by composing the corresponding representations with the homomorphism $G \rightarrow G_{0}$ given by $g_{0} n^{*} \rightarrow g_{0}$, together with a single extra character $\chi_{l+1}$ which is $\rho-1$, where $\rho$ is the permutation character corresponding to the representation of $G$ as a permutation group on $F$. Thus

$$
\begin{aligned}
\chi_{i}\left(g_{0} n^{*}\right) & =\bar{\chi}_{i}\left(g_{0}\right), & & i=1, \ldots, l, g_{0} \neq e, g_{0} \in G_{0}, n^{*} \in N, \\
\chi_{i}\left(n^{*}\right) & =\chi_{i}(e), & & i=1, \ldots, l, n^{*} \in N, \\
\chi_{l+1}(e) & =p^{n}-1, & & \\
\chi_{l+1}\left(g_{0} n^{*}\right) & =0 & & \text { if } g_{0} \neq e, \\
\chi_{l+1}\left(n^{*}\right) & =-1 & & \text { if } n^{*} \neq e \in N .
\end{aligned}
$$

We claim that the conjugacy classes of $G$ are of the following form. Let $C l_{G}(g)$ denote the conjugacy class of the element $g$ in $G$. Then if $e \neq g_{0} \in G_{0}$, it follows that $C l_{G}\left(g_{0}\right)=\left\{x n^{*} ; x \in C l_{G_{0}}(g), n^{*} \in N\right\}$. The remaining two classes are $\{e\}$ and $N-\{e\}$. Suppose $g_{0} \neq e$ lies in $G_{0}$. An arbitrary element $y \in G$ may be written $y=y_{0} n^{*}, y_{0} \in G_{0}, n^{*} \in N$. Then

$$
\begin{aligned}
g_{0}^{y} & =\left(g_{0}^{y_{0}}\right)^{n^{*}}=\left(n^{*}\right)^{-1} g_{0}^{\prime} n^{*} \quad\left(g_{0}^{\prime}=g_{0}^{y_{0}} \in G_{0}\right) \\
& =g_{0}^{\prime}\left(n^{*}\right)^{-1} g_{0}^{\prime} n^{*}=g_{0}^{\prime} n^{\prime *} \quad\left(\text { for some } n^{\prime} \in N\right) .
\end{aligned}
$$

Now suppose $g_{0}^{x}=g_{0}$ for $x=x_{0} n^{*} \in G$. Then $g_{0}=n^{*-1} g_{0}^{x_{0}} n^{*}$. But since $G$ is Frobenius, $\left(n^{*}\right)^{-1} g_{0}^{x_{0}} n^{*}$ fixes 0 if and only if $n^{*}=e$. Hence $C_{G}\left(g_{0}\right)=C_{G_{0}}\left(g_{0}\right)$. Thus $\left[G: C_{G}\left(g_{0}\right)\right]=\left[G: G_{0}\right]\left[G_{0}: C_{G_{0}}\left(g_{0}\right)\right]$, i.e., 
$\left|C l_{G}\left(g_{0}\right)\right|=|N|\left|C l_{G_{0}}\left(g_{0}\right)\right|$ and hence $C l_{G}\left(g_{0}\right)=\left\{x n^{*} ; n^{*} \in N, x \in C l_{G_{0}}\left(g_{0}\right)\right\}$. Since $C_{G}\left(n^{*}\right)=N-\{e\}$ for $n^{*} \in N-\{e\}$, the above claim is justified.

We now set up the correspondence between the characters of $G_{0}$ and $H_{0}$, $\bar{\chi}_{i} \leftrightarrow \bar{\mu}_{i}$, by means of the isomorphism $\theta$ :

$$
\bar{\chi}_{i}\left(g_{0}\right)=\bar{\mu}_{i}\left(\theta\left(g_{0}\right)\right), \quad g_{0} \in G_{0} .
$$

The characters $\mu_{1}, \ldots, \mu_{l}$ of $H$ are defined by

$$
\mu_{i}\left(h_{0} n^{*}\right)=\bar{\mu}_{i}\left(h_{0}\right), \quad i=1, \ldots, l,
$$

and again $\mu_{l+1}=\rho-1$. Thus

$$
\chi_{i}\left(g_{0} n^{*}\right)=\mu_{i}\left(\theta\left(g_{0}\right)\left(n^{q}\right)^{*}\right), \quad i=1, \ldots, l+1,
$$

and

$$
\chi_{i}(g)=\mu_{i}(\psi(g)), \quad i=1, \ldots, l+1, g \in G .
$$

We now verify that (1.3) is satisfied by all possible choices for $\nu$.

$$
\begin{aligned}
\nu=\chi_{i} \circ \chi_{j}, \quad \tau & =\mu_{i} \circ \mu_{j}, \quad 1 \leq i<j \leq l+1, \\
\nu\left(g, g^{\prime}\right) & =\chi_{i}(g) \chi_{j}\left(g^{\prime}\right)+\chi_{i}\left(g^{\prime}\right) \chi_{j}(g) \\
& =\mu_{i}(\psi(g)) \mu_{j}\left(\psi\left(g^{\prime}\right)\right)+\mu_{i}\left(\psi\left(g^{\prime}\right)\right) \mu_{j}(\psi(g)) \\
& =\tau\left(\psi(g), \psi\left(g^{\prime}\right)\right) .
\end{aligned}
$$

$$
\nu=\chi_{j}^{(2)} \text { or } \chi_{j}^{(2,+)}, \quad 1 \leq j \leq l .
$$

If $\nu=\chi_{j}^{(2)}$, then $\nu\left(g, g^{\prime}\right)=\chi_{j}(g) \chi_{j}\left(g^{\prime}\right)-\chi_{j}\left(g g^{\prime}\right)$. Let $g=g_{0} n^{*}$ and $g^{\prime}=g_{0}^{\prime} n^{\prime *}$. Then $g h=g_{0} g_{0}^{\prime} n^{\prime \prime *}$ for some $n^{\prime \prime} \in N$. Hence

$$
\begin{aligned}
\nu\left(g, g^{\prime}\right) & =\chi_{j}(\psi(g)) \chi_{j}\left(\psi\left(g^{\prime}\right)\right)-\bar{\chi}_{j}\left(g_{0} g_{0}^{\prime}\right) \\
& =\mu_{j}(\psi(g)) \mu_{j}\left(\psi\left(g^{\prime}\right)\right)-\bar{\mu}_{j}\left(\theta\left(g_{0} g_{0}^{\prime}\right)\right) \\
& =\mu_{j}(\psi(g)) \mu_{j}\left(\psi\left(g^{\prime}\right)\right)-\mu_{j}\left(\psi\left(g_{0}\right) \psi\left(g_{0}^{\prime}\right)\right) \\
& =\tau\left(\psi(g), \psi\left(g^{\prime}\right)\right) .
\end{aligned}
$$

The second case is similar (note that $\chi_{j}^{(2)}$ occurs as a 2-character only if $\left.\operatorname{deg}\left(\chi_{j}\right) \geq 2\right)$.

$$
\nu=\chi_{l+1}^{(2)} \text { or } \chi_{l+1}^{(2,+)}
$$

let $\nu=\chi_{l+1}^{(2)}$. For convenience we omit the suffix $l+1$, thus

$$
\nu\left(g, g^{\prime}\right)=\chi(g) \chi\left(g^{\prime}\right)-\chi\left(g g^{\prime}\right) \text {. }
$$

Let $g=g_{0} n^{*}$ and $g^{\prime}=g_{0}^{\prime} n^{\prime *}$.

We consider two cases:

Case 1. $g_{0} g_{0}^{\prime} \neq e$. Then $\chi\left(g g^{\prime}\right)=\chi\left(g_{0} g_{0}^{\prime} n^{\prime \prime *}\right)=0$ and

$$
\begin{aligned}
\psi(g) \psi\left(g^{\prime}\right) & =\theta\left(g_{0}\right)\left(n^{q}\right)^{*} \theta\left(g_{0}^{\prime}\right)\left(n^{\prime q}\right)^{*} \\
& =\theta\left(g_{0}\right) \theta\left(g_{0}^{\prime}\right) n^{\prime \prime \prime *} \quad\left(n^{\prime \prime \prime *} \in N\right) \\
& =\theta\left(g_{0} g_{0}^{\prime}\right) n^{\prime \prime \prime *} .
\end{aligned}
$$

So $\psi(g) \psi\left(g^{\prime}\right)$ is not an element of $N$. Hence $\mu\left(\psi(g) \psi\left(g^{\prime}\right)\right)=0$. Thus $\nu\left(g, g^{\prime}\right)=\chi(g) \chi\left(g^{\prime}\right)=\mu(\psi(g)) \mu\left(\psi\left(g^{\prime}\right)\right)=\tau\left(\psi(g), \psi\left(g^{\prime}\right)\right)$. 
Case 2. $g g^{\prime} \in N$. We show that $g g^{\prime}=e$ if and only if $\psi(g) \psi\left(g^{\prime}\right)=e$. It will then follow that

$$
\chi\left(g g^{\prime}\right)=\mu\left(\psi(g) \psi\left(g^{\prime}\right)\right) \text { for all } g, g^{\prime} \text { such that } g g^{\prime} \in N \text {, }
$$

and as in Case 1 that $\nu\left(g, g^{\prime}\right)=\lambda\left(\psi(g), \psi\left(g^{\prime}\right)\right)$. Thus suppose $g_{0} g_{0}^{\prime}=e$. Then

$$
g g^{\prime}=g_{0} g_{0}^{\prime}\left(n^{*}\right)^{g_{0}^{\prime}} n^{\prime *}=\left(n^{*}\right)^{g_{0}^{\prime}} n^{\prime *} \text {. }
$$

Now let $g_{0}^{\prime}=\sigma^{v t} \bar{\omega}^{\lambda}$. It follows from Lemma 1.2 in [2] that

$$
\left(n^{*}\right)^{g_{0}^{\prime}}\left(n^{\prime *}\right)=\left(n^{p^{v t}} \omega^{\lambda}\right)^{*}\left(n^{\prime}\right)^{*}=\left(n^{p^{v t}} \omega^{\lambda}+n^{\prime}\right)^{*}
$$

and thus $g g^{\prime}=e$ if and only if $n p^{v t} \omega^{\lambda}+n^{\prime}=0$, i.e., if and only if $-n^{p^{v}} \omega^{\lambda}=n^{\prime}$. Now

$$
\begin{aligned}
\psi(g) \psi\left(g^{\prime}\right) & =\theta\left(g_{0}\right)\left(n^{q}\right)^{*} \theta\left(g_{0}^{\prime}\right)\left(n^{\prime q}\right)^{*} \\
& =\theta\left(g_{0}\right) \theta\left(g_{0}^{\prime}\right)\left(n^{q}\right)^{* \theta\left(g_{0}^{\prime}\right)}\left(n^{\prime q}\right)^{*} \\
& =\left(n^{q}\right)^{* \theta\left(g_{0}^{\prime}\right)}\left(n^{\prime q}\right)^{*}=\left(n^{q}\right)^{* \sigma^{v t} \omega^{\lambda q}}\left(n^{\prime q}\right)^{*} \\
& =\left(n^{q p^{v t}} \omega^{\lambda q}\right)^{*}\left(n^{\prime q}\right)^{*}=\left(n^{q p^{t}} \omega^{\lambda q}+n^{\prime q}\right)^{*}
\end{aligned}
$$

which is $e$ if and only if $-n^{q p^{v t}} \omega^{\lambda q}=n^{\prime q}$. Thus $g g^{\prime}=e$ if and only if $\psi(g) \psi\left(g^{\prime}\right)=e$.

The case $v=\chi^{(2,+)}$ is similar.

Hence in all cases we have shown that (1.3) holds; i.e., the 2-character tables of $G$ and $H$ are the same.

Proof that $(\mathrm{b}) \Rightarrow(\mathrm{a})$. This is immediate on noting that

(i) If $G$ and $H$ have the same 2-character tables then they necessarily have the same ordinary character tables (see $\S 1$ ).

(ii) As quoted above, in [2] it is shown that if $G$ is any doubly transitive solvable group and $H$ has the same ordinary character table as $G$ then $\{G, H\}$ form a Brauer pair.

Example. Suppose $p=5, n=4, k=4, v=1, i=1$, and $j=3$. Then $G$ and $H$ are nonisomorphic groups of order $624 \cdot 625$. By Theorem 2.1, $G$ and $H$ have the same 2-character tables. An explicit value for $q$ in this case is 7 .

\section{SOME OPEN PROBLEMS}

A consequence of the work in [5] is that if a representation is sufficiently large its 3-character is sufficient to determine the group. There remains the question of how much information the 3-character of an arbitrary faithful representation contains. In [8] the case of an irreducible representation of degree 2 is considered. Here the 2-character alone contains sufficient information to construct an explicit matrix representation. Thus the 2-character of a faithful irreducible representation of degree 2 determines the group.

Problem 1. Let $\chi$ be a faithful irreducible representation of $G$ of degree greater than 2. Does $\chi^{(3)}$ determine $G$ ?

By the results of $\S 2$, the condition that $G$ and $H$ have the same 2-character tables is not sufficient to ensure that $G$ and $H$ are isomorphic. Since Brauer pairs have been the subject of investigation, we pose the following. 
Problem 2. If $G$ and $H$ have the same 2-character table must $G$ and $H$ necessarily form a Brauer pair?

Finally we consider representations over fields of finite characteristic. By [4], the group determinant over any field whose characteristic does not divide $|G|$ determines $G$. In [5] it is shown that if $\operatorname{char}(K) \neq 2$ and $\operatorname{char}(K) \nmid|G|$ the 1-, 2 -, and 3-characters of the regular representation over the field $K$ determine $G$.

Problem 3. Let $G$ be a group of odd order and $K$ be a field of characteristic 2. Which is the smallest value of $k$ for which the $1-, 2-, \ldots, k$-characters of the regular representation over $K$ determine $G$ ?

\section{REFERENCES}

1. R. Brauer, Representations of finite groups, Lectures in Modern Mathematics (T. L. Saaty, ed.), vol. 1, Wiley, New York, 1963, pp. 133-175.

2. G. Cliff and S. Sehgal, On groups having the same character tables, Comm. Algebra 9 (1981), 627-640.

3. G. Frobenius, Über die Primfaktoren der Gruppendeterminante, Sber. Akad. Wiss. Berlin (1896), 1343-1382.

4. E. Formanek and D. Sibley, The group determinant determines the group, Proc. Amer. Math. Soc. 112 (1991), 649-656.

5. H. J. Hoehnke and K. W. Johnson, The 3-characters are sufficient for the group determinant, Proc. Ring Theory Conference (Barnaul, Siberia), 1991 (to appear).

6. B. Huppert, Zweifach transitive, auflosbäre Permutationgruppen, Math. Z. 68 (1957), 126150.

7. K. W. Johnson, Latin square determinants, Algebraic, Extremal and Metric Combinatorics 1986, London Math. Soc. Lecture Notes Ser., vol. 131, Cambridge Univ. Press, London and New York, 1988, pp. 146-154.

8. __ On the group determinant, Math. Proc. Cambridge Philos. Soc. 109 (1991), 299-311.

9. R. Mansfield, A group determinant determines its group, preprint.

Department of Mathematics, The Pennsylvania State University, Abington, PennsylVANIA 19001

E-mail address: kwj1@psuvm.bitnet

Department of Mathematics, The Ohio State University, Columbus, Ohio 43210

E-mail address: sehgal@math.ohio-state.edu 